\title{
BTA 深孔精密扩孔系统流体扰动的非线性横振
}

\author{
赵 武 ${ }^{1}$ 霍博义 $^{1,2,3}$ 黄 丹 ${ }^{2}$ \\ (1. 河南理工大学机械与动力工程学院 焦作 454000 ; \\ 2. 河南理工大学材料科学与工程学院 焦作 454000; \\ 3. 上海振华重工（集团）股份有限公司 上海 200125)
}

\begin{abstract}
摘要: 为提高超大长径比超临界汽轮机转子内孔的加工精度, 研究了高速 BTA 深孔精密扩孔工艺中的钻杆(刀轴)横向振动问 题。通过建立以流体扰动为主要诱因的深孔钻杆系统的非线性横向振动模型, 运用 Melnikov 方法分析了流体扰动下系统横 向非线性振动的混沌性态; 利用图胞映射理论研究多初值条件下的流体扰动对 BTA 深孔加工系统安全盆域中吸引子和吸引 域的流形变化, 明确了在多初值条件下, 该系统吸引子的运动特性。结合分岔、最大 Lyapunov 指数、双参平面分岔等特征, 分析了流体扰动激励下 BTA 深孔切削系统随各类参数变化的非线性动力行为, 通过双参平面分岔分析, 讨论了该系统在双 参变化时的非线性动力特性, 揭示了 BTA 深孔加工系统在单参、双参变化时的非线性动力行为演变机制, 并对系统横向振 动的混沌参数域进行了解析, 得到系统在双参数平面上混沌运动、稳定周期运动的参数域。该研究结果可为 BTA 深孔切削 加工的生产实践提供一定理论指导。
\end{abstract}

关键词: BTA 深孔加工; 横向振动; 分岔; 吸引子; 最大 Lyapunov 指数; 双参分岔

中图分类号: TH161; TH113

\section{Nonlinear Transverse Vibration Induced by Fluid Disturbance on BTA Deep Hole Precision Reaming System}

\author{
ZHAO Wu ${ }^{1}$ HUO Boyi ${ }^{1,2,3}$ HUANG Dan ${ }^{2}$
}

(1. School of Mechanical and Power Engineering, Henan Polytechnic University, Jiaozuo 454000;

2. School of Materials Science and Engineering, Henan Polytechnic University, Jiaozuo 454000;

3. Shanghai Zhenhua Port Machinery Company Limited, Shanghai 200125)

\begin{abstract}
In order to promote the machining precision of the inner hole in super-critical turbine rotor with super length-diameter ratio, the transverse vibration on BTA deep-hole precision reaming bar (cutter shaft) is studied. Based on the established nonlinear transverse vibration model for the deep-hole reaming system, where the oscillation is mainly induced by the fluid disturbance, the chaos of the systematic nonlinear transverse vibration induced by fluid disturbance is analyzed with Melnikov method; by way of the cell mapping theory, under multiple initial values, it is studied for the effects of the fluid disturbance on the manifold deformation of the attractor and attraction domain in the safe basin domain in the BTA deep-hole reaming system, and it is demonstrated for the motion characteristic of the attractor in system. Combined with the bifurcation, maximum Lyapunov exponent, as well as the bifurcation on double parameters plane, it is analyzed for the nonlinear dynamic behavior of the BTA deep hole reaming system which is varied with kinds of parameters. In addition, through the analysis of the bifurcation of bi-parameter plane, the nonlinear dynamic behavior in the system is discussed at the variation of the bi-parameter, and the evolvement mechanism of the nonlinear dynamic behaviors is revealed at the variation both of single and double parameters in the BTA system. At last, the chaos parameters is analyzed for the systematic transverse vibration and the parameters domain in the system is obtained for both the chaos motion on bi-parameter plane and stable periodic motion. The results would provide some useful theoretical guide for the practice in BTA deep hole reaming.
\end{abstract}

Key words: deep-hole reaming system; transverse vibration; bifurcation; attractor; maximum Lyapunov exponent; double parameter bifurcation

* 国家自然科学基金(51075126)、河南省培育资助(NSFRF170503)、河南 省科技攻关资助(192102210052)和创新团队资助(T2019-5)资助项目。

20191220 收到初稿, 20200415 收到修改稿 


\section{0 前言}

超临界汽轮机转子内孔全长 $9600 \mathrm{~mm}$, 综合跳 动 $5 \mu \mathrm{m}$, 表面粗䊁度 $0.4 \mu \mathrm{m}$ 。当以高速 BTA 深孔 精加工完成扩孔工艺, 可避免加工过程内孔壁的应 力集中对转子在服役过程中残余应力释放的影响。 该工艺实施中，钻杆长径比高达 180 200, 深孔加 工的排屑、冷却、导向三大矛盾尤为突出, 需要大 量的切削液来改善冷却、润滑以及排屑的效率。旋 转的钻杆引起钻杆内、外切削液扰动, 对钻杆的横 向运动精度产生很大影响。胡占齐等 ${ }^{[1-2]}$ 推导了钻杆 旋转时切削液对钻杆扰动的反馈力表达式, 通过建 立含有切削液扰动的钻杆横向振动模型 ${ }^{[3-5]}$, 对切削 液诱发钻杆浴动做了深入研究, 表明切削液扰动对 BTA 深孔加工非线性振动的影响不容忽视。这为进 一步研究切削液扰动下, BTA 深孔切削系统的非线 性振动奠定了基础。

对细长杆梁非线性横向振动研究中, 罗忠等 ${ }^{[6]}$ 综述了动力学相似理论在结构动力学相似试验模型 设计中的应用研究进展与发展趋势, 总结了动力学 相似理论在大型动力装备动力学相似试验模型设计 中的应用以及转子系统的动力学相似试验模型设计 方法。丁虎等 ${ }^{[7]}$ 以积分-偏微分方法得到轴向运动梁 的横向非线性模型，仿真结果表明该模型比其它模 型计算耦合振动的精度高。韦娟等 ${ }^{[8]}$ 对直圆柱形谐 振管内的非线性驻波进行数值仿真计算, 为实现谐 振管内非线性驻波的工程应用奠定了良好的基础。 金基铎等 ${ }^{[9]}$ 建立了固定简支细长圆管在横流作用下 的非线性横振模型, 并分析了流速对系统分岔、颤 振等特性的影响。XU 等 ${ }^{[10-13]}$ 系统地运用图胞映射 法分析了 Duffing 系统吸引子、吸引域的形态。

BYKADOR 等 ${ }^{[14]}$ 通过研究深孔加工孔表面与 钻头接触作用力的影响, 解释了深孔切削过程非 线性分岔的形成机理, 从一定程度上揭示了刀具 失效和典型缺陷的发展过程。CHEN 等 ${ }^{[15]}$ 把 BTA 深孔刀具系统看作以钻杆轴为主体的二阶质量动 力系统的伯努利-欧拉型梁模型, 采用组合解析法 和有限元法求解了横向和纵向运动的解, 并比较 了钻轴由祄套和支架错位引起的偏差对钻孔直线 度的影响, 试验结果表明, 有限元模型对直线度 误差的预测精度较差。

WEINERT K 等 ${ }^{[16]}$ 研究了大长径比 BTA 深孔钻 削扭转颤振的影响。通过对比无振和扭振模态, 明 确了扭振对系统模态阻尼的深度依赖。张洁等 ${ }^{[17]}$ 针 对柔性双自由度铣削工艺系统, 基于刚体运动学
的静态切削力成分和再生效应的动态切削力成分, 建立了时滞微分动力学模型, 预测了无颤振工艺 参数区域。孔令飞等 ${ }^{[18]}$ 深孔钻削所产生的切削热 是引起加工孔表面损伤和热变形的主要原因，依 据 BTA 深孔钻削工艺的特点, 提出一种基于深孔 制件多点温度的时空信息反演计算切削热通量分 布特征的方法。

本文通过建立 BTA 精密扩孔工序中以流体扰 动为主要诱因的深孔钻杆系统的非线性横向振动模 型, 运用图胞映射法研究多初值条件下的流体扰动 对 BTA 深孔钻杆系统的吸引子运动特性。通过最大 Lyapunov 指数、双参数平面分岔等特性, 揭示了 BTA 深孔加工系统在单参、双参变化时的非线性动 力行为演变机制, 并对系统横向振动的混沌参数域 进行了解析，该工作可为工程应用提供理论指导。

\section{BTA 深孔切削系统横向振动模型}

深孔切削加工系统如图 1 所示。在 BTA 精密扩 孔的深孔加工中, 系统的横向激励应为横向硬态切 削力和切削液横向扰动反馈力之和。但在精孔扩孔 工序中，切深量和进给量都很小，则硬态切削力对 系统的扰动影响较小, 所以该工况下系统的横向激 励扰动主要来源于该工艺系统中钻杆(刀具轴)受到 的内、外切削液的扰动。

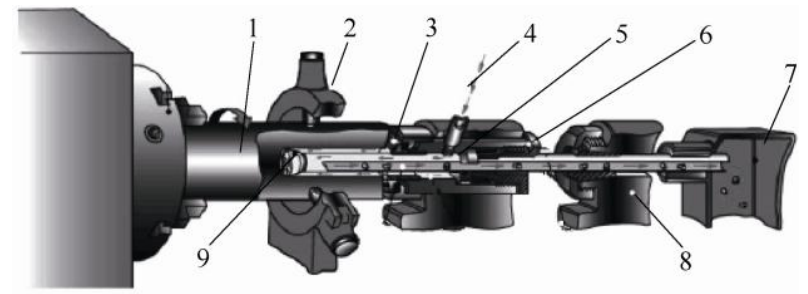

图 1 BTA 深孔切削系统示意图

1.工件 2 .支撑架 3 .动力封头 4 . 切削液导管 5 . 钻杆支撑套 6.密封套 7.排首口 8.钻杆支撑架 9.刀具

为探究流体扰动下 BTA 深孔切削系统的非线 性全局特性, 考虑切削系统切削液扰动的横向反馈 力, 建立 BTA 深孔切削系统横向非线性振动模型, 其 $y-x$ 方向上简化的微元受力分析模型如图 2 所示。

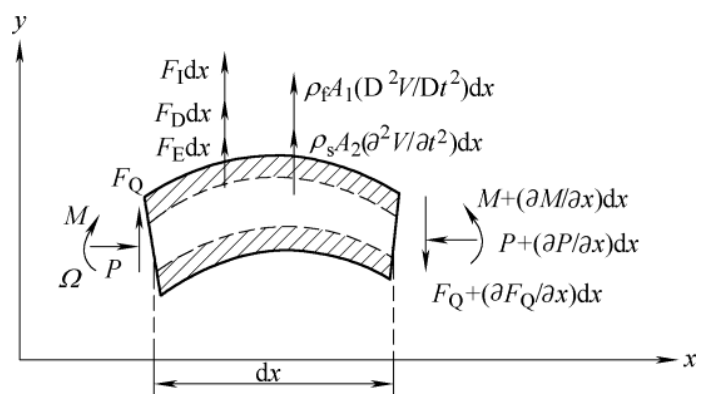

图 2 BTA 深孔钻杆微元受力分析模型 
在深孔钻杆上任取一微元 $\mathrm{d} x$ 建立横向振动微 分方程。假定深孔钻杆的振动为平面振动, 则变形 仅发生在 $y-x$ 平面内, 如图 2 所示: $\rho_{\mathrm{f}}$ 是切削液密 度, $\rho_{\mathrm{s}}$ 是钻杆的材料密度, $A_{1} 、 A_{2}$ 分别为钻杆内孔 横截面积、钻杆横截面积, $F_{\mathrm{Q}}$ 是剪切力, $M$ 是弯矩, $P$ 是钻杆轴向力, $F_{\mathrm{D}} \mathrm{d} x$ 是科氏力, $F_{\mathrm{D}}=2 \rho I \Omega\left(\partial^{3} W /\right.$ $\left.\partial x^{2} \partial t\right), \rho_{\mathrm{s}} A_{2}\left(\partial^{2} V / \partial t^{2}\right) \mathrm{d} x$ 是深孔镗杆微元的惯性力, $F_{\mathrm{I}} \mathrm{d} x$ 是钻杆旋转惯量效应力, $F_{\mathrm{I}}=\rho_{I}\left(\partial^{4} V / \partial x^{2} \partial t^{2}\right), F_{\mathrm{E}} \mathrm{d} x$ 是旋转钻杆向心力, $F_{\mathrm{E}}=\rho_{I} \Omega^{2}\left(\partial^{2} V / \partial x^{2}\right)$ 。 $W(x, t) 、 V(x$, $t$ )分别为任意时刻 BTA 深孔钻杆在 $y 、 z$ 方向的径向 位移。

钻杆内孔中单位长度质量为 $\rho_{\mathrm{f}} A_{1}$ 的流体以流速 $v_{1}$ 流过, 则钻杆在旋转过程中 $y$ 方向上的流体惯性 力 $\rho_{\mathrm{f}} A_{1}\left(\partial^{2} V / \partial t^{2}\right)$ 可表示为

$$
\begin{gathered}
\rho_{\mathrm{f}} A_{1} \frac{D^{2} V}{D t^{2}}=\rho_{\mathrm{f}} A_{1}\left(\frac{\partial}{\partial t}+v_{1} \frac{\partial}{\partial x}\right)^{2} V= \\
\rho_{\mathrm{f}} A_{1} \frac{\partial^{2} V}{\partial t^{2}}+2 \rho_{\mathrm{f}} A_{1} v_{1} \frac{\partial^{2} V}{\partial x \partial t}+\rho_{\mathrm{f}} A_{1} v_{1}^{2} \frac{\partial^{2} V}{\partial x^{2}}
\end{gathered}
$$

式(1)右边各项依次分别是流体牵连惯性力、流体科 氏惯性力、流体相对惯性力。

由图 2 模型建立 $y-x$ 平面内深孔钻杆微分方程

$$
\left\{\begin{array}{l}
\frac{\partial P}{\partial x}=0 \\
\frac{\partial F_{\mathrm{Q}}}{\partial x}-\rho_{\mathrm{f}} A_{1} \frac{D^{2} V}{D t^{2}}-\rho_{\mathrm{s}} A_{2} \frac{\partial^{2} V}{\partial t^{2}}-F_{\mathrm{D}}-F_{\mathrm{I}}-F_{\mathrm{E}}=0 \\
\frac{\partial M}{\partial x}-F_{\mathrm{Q}}-P \frac{\partial V}{\partial x}=0
\end{array}\right.
$$

解析式(2)内相关力与变形、位移导数的关系

$$
\begin{gathered}
M=-E I \frac{\partial^{2} V}{\partial x^{2}} \\
F_{\mathrm{Q}}=\frac{\partial M}{\partial x}-P \frac{\partial V}{\partial x}=-E I \frac{\partial^{3} V}{\partial x^{3}}-P \frac{\partial^{2} V}{\partial x^{2}}
\end{gathered}
$$

式中, $E$ 为拉压弹性模量; $I 、 \Omega$ 分别为 BTA 深孔 钻杆截面惯性矩、旋转角速度。

联立式(2)、(3), 可得 BTA 深孔切削系统 $y-x$ 平面内横向振动微分方程

$$
\begin{gathered}
E I \frac{\partial^{4} V}{\partial x^{4}}+\left(P-\rho_{\mathrm{s}} I \Omega^{2}-\rho_{\mathrm{f}} A_{1} v_{1}^{2}\right) \frac{\partial^{2} V}{\partial x^{2}}- \\
\left(\rho_{\mathrm{f}} A_{1}+\rho_{\mathrm{s}} A_{2}\right) \frac{\partial^{2} V}{\partial t^{2}}-\rho_{\mathrm{s}} I \frac{\partial^{4} V}{\partial x^{2} \partial t^{2}}- \\
2 \rho_{\mathrm{s}} I \Omega \frac{\partial^{3} W}{\partial x^{2} \partial t}-2 \rho_{\mathrm{f}} A_{1} v_{1} \frac{\partial^{2} V}{\partial x \partial t}=0
\end{gathered}
$$

同理可得 BTA 深孔切削系统 $z-x$ 平面内横向振动微 分方程为

$$
\begin{gathered}
E I \frac{\partial^{4} W}{\partial x^{4}}+\left(P-\rho_{\mathrm{s}} I \Omega^{2}-\rho_{\mathrm{f}} A_{1} v_{1}^{2}\right) \frac{\partial^{2} W}{\partial x^{2}}- \\
\left(\rho_{\mathrm{f}} A_{1}+\rho_{\mathrm{s}} A_{2}\right) \frac{\partial^{2} W}{\partial t^{2}}-\rho_{\mathrm{s}} I \frac{\partial^{4} W}{\partial x^{2} \partial t^{2}}- \\
2 \rho_{\mathrm{s}} I \Omega \frac{\partial^{3} V}{\partial x^{2} \partial t}-2 \rho_{\mathrm{f}} A_{1} v_{1} \frac{\partial^{2} W}{\partial x \partial t}=0
\end{gathered}
$$

式(4)、式(5)振动微分方程的边界条件为

$$
\begin{array}{cc}
V(0, t)=\frac{\partial^{2} V}{\partial x^{2}}(0, t)=0 & V(L, t)=\frac{\partial^{2} V}{\partial x^{2}}(L, t)=0 \\
W(0, t)=\frac{\partial^{2} W}{\partial x^{2}}(0, t)=0 & W(L, t)=\frac{\partial^{2} W}{\partial x^{2}}(L, t)=0
\end{array}
$$

初始条件为

$$
\begin{array}{lll}
V(x, 0)=a \sin \left(\frac{m \pi x}{L}\right) & \frac{\partial V}{\partial x}(x, 0)=0 & m=1,2, \cdots \\
W(x, 0)=b \sin \left(\frac{m \pi x}{L}\right) & \frac{\partial V}{\partial x}(x, 0)=0 & m=1,2, \cdots
\end{array}
$$

式中, $a 、 b$ 分别为 $x 、 y$ 方向上振幅, 假设钻杆在 任意时刻在 $V 、 W$ 方向上的位移为

$$
\begin{gathered}
V(x, t)=a v(t) \sin \left(\frac{m \pi x}{L}\right) \quad m=1,2, \cdots \\
W(x, t)=b w(t) \sin \left(\frac{m \pi x}{L}\right) \quad m=1,2, \cdots
\end{gathered}
$$

则 BTA 钻杆的轴向缩短量为

$$
\delta_{1}=\frac{1}{2} \int_{0}^{L}\left(\frac{\partial V}{\partial x}\right)^{2} \mathrm{~d} x \quad \delta_{2}=\frac{1}{2} \int_{0}^{L}\left(\frac{\partial W}{\partial x}\right)^{2} \mathrm{~d} x
$$

由拉压胡克定律知

$$
\delta_{1}=\Delta P_{1} L / E A_{2} \quad \delta_{2}=\Delta P_{2} L / E A_{2}
$$

式中 $\Delta P_{1}-V$ 方向上附加轴向拉力;

$\Delta P_{2}-W$ 方向上附加轴向拉力;

$L$ 一钻杆的长度。

由式(9)和式(10)的附加轴向拉力大小为

$$
\begin{aligned}
& P_{1}=P_{0}-\frac{E A_{2}}{2 L} \int_{0}^{L}\left(\frac{\partial V}{\partial x}\right)^{2} \mathrm{~d} x=P_{0}-\frac{E a^{2} v^{2} \pi^{2} m^{2} A_{2}}{4 L^{2}} \\
& P_{2}=P_{0}-\frac{E A_{2}}{2 L} \int_{0}^{L}\left(\frac{\partial W}{\partial x}\right)^{2} \mathrm{~d} x=P_{0}-\frac{E b^{2} w^{2} \pi^{2} m^{2} A_{2}}{4 L^{2}}
\end{aligned}
$$

设 $P_{1} 、 P_{2}$ 分别 $y 、 z$ 方向的轴向总压力为

$$
\begin{aligned}
& P_{1}=P_{0}-\frac{E A_{2}}{2 L} \int_{0}^{L}\left(\frac{\partial V}{\partial x}\right)^{2} \mathrm{~d} x=P_{0}-\frac{E a^{2} v^{2} \pi^{2} m^{2} A_{2}}{4 L^{2}} \\
& P_{2}=P_{0}-\frac{E A_{2}}{2 L} \int_{0}^{L}\left(\frac{\partial W}{\partial x}\right)^{2} \mathrm{~d} x=P_{0}-\frac{E b^{2} w^{2} \pi^{2} m^{2} A_{2}}{4 L^{2}}
\end{aligned}
$$

式中, $P_{0}$ 为钻杆的初始轴向力。

这里假设 $v(t)$ 为钻杆关于时间的函数。将式(6)、 
(7)、(8)、(12)代入到式(4)、(5)中, 经运算整理得到 $y 、 z$ 方向耦合的非线性方程为

$$
\begin{gathered}
\left(\rho_{\mathrm{f}} A_{1}+\rho_{\mathrm{s}} A_{2}-\rho_{\mathrm{s}} I \frac{\pi^{2} m^{2}}{L^{2}}\right) a \ddot{v}-\left(2 \rho_{\mathrm{s}} I \Omega+2 \rho_{\mathrm{f}} A_{1} v_{1}\right) \times \\
b \frac{\pi^{2} m^{2}}{L^{2}} \dot{w}-\left[\left(P_{0}-\rho_{\mathrm{s}} I \Omega^{2}-\rho_{\mathrm{f}} A_{1} v_{1}^{2}\right) \frac{\pi^{2} m^{2}}{L^{2}}-E I \frac{\pi^{2} m^{2}}{L^{2}}\right] \times \\
a v+\frac{E a^{3} \pi^{2} m^{2} A_{2}}{4 L^{2}} v^{3}=0 \\
\left(\rho_{\mathrm{f}} A_{1}+\rho_{\mathrm{s}} A_{2}-\rho_{\mathrm{s}} I \frac{\pi^{2} m^{2}}{L^{2}}\right) a \ddot{w}-\left(2 \rho_{\mathrm{s}} I \Omega+2 \rho_{\mathrm{f}} A_{1} v_{1}\right) \times \\
b \frac{\pi^{2} m^{2}}{L^{2}} \dot{v}-\left[\left(P_{0}-\rho_{\mathrm{s}} I \Omega^{2}-\rho_{\mathrm{f}} A_{1} v_{1}^{2}\right) \frac{\pi^{2} m^{2}}{L^{2}}-E I \frac{\pi^{2} m^{2}}{L^{2}}\right] \\
a w+\frac{E a^{3} \pi^{2} m^{2} A_{2}}{4 L^{2}} w^{3}=0
\end{gathered}
$$

令

$$
\begin{gathered}
\gamma=\rho_{\mathrm{f}} A_{1}+\rho_{\mathrm{s}} A_{2}-\rho_{\mathrm{s}} I \frac{\pi^{2} m^{2}}{L^{2}} \\
\eta_{1}=-\left(\rho_{\mathrm{s}} I \Omega+\rho_{\mathrm{f}} A_{1} v_{1}\right) \frac{\pi^{2} m^{2}}{L^{2}} \\
\kappa_{1}=\left(P_{0}-\rho_{\mathrm{s}} I \Omega^{2}-\rho_{\mathrm{f}} A_{1} v_{1}^{2}\right) \frac{\pi^{2} m^{2}}{L^{2}}-E I \frac{\pi^{2} m^{2}}{L^{2}} \\
\lambda_{1}=\frac{E a^{2} \pi^{2} m^{2} A_{2}}{4 L^{2}}
\end{gathered}
$$

则式(13)、式(14)整理为

$$
\begin{gathered}
\gamma a \ddot{v}+2 \eta_{1} b \dot{v}-\kappa_{1} a v+\lambda_{1} a v^{3}=0 \\
\gamma b \ddot{w}-2 \eta_{1} a \dot{w}-\kappa_{1} b w+\lambda_{1} b w^{3}=0
\end{gathered}
$$

令 $u=v-w$, 式(15)减去式(16)得

$\gamma(a-b) \ddot{u}+2 \eta_{1}(b+a) \dot{u}-\kappa_{1}(a-b) u+\lambda_{1}(a-b) u^{3}=0$

将式(17)量纲一化得

$$
\ddot{u}+2 \eta \dot{u}-\kappa u+\lambda u^{3}=0
$$

其中

$$
\eta=\frac{\eta_{1}(a+b)}{\gamma(a-b)} ; \kappa=\frac{\kappa_{1}}{\gamma} ; \lambda=\frac{\lambda_{1}}{\gamma}
$$

把 BTA 深孔切削系统切削液的横向反馈力变 化描述为

$$
F=F_{1}+F_{2} \sin \left(\omega_{\mathrm{b}} t\right)
$$

则式(18)变为

$$
\ddot{u}+2 \eta \dot{u}-\kappa u+\lambda u^{3}=F_{1}+F_{2} \sin \left(\omega_{\mathrm{b}} t\right)
$$

式中, $\eta$ 为量纲一阻尼比; $\kappa$ 为量纲一一次方刚度比; $\lambda$ 为量纲一立方刚度比; $F_{1}$ 为切削液扰动的反馈力 均值; $F_{2}$ 为切削液扰动的反馈力波动值; $\omega_{\mathrm{b}}$ 为系统
频率与切削液扰动的横向反馈力波动频率比。

则式(20)所代表的 BTA 深孔切削系统的强迫 振动模型如图 3 所示。其中, $K$ 为钻杆横向振动 刚度; $c$ 为钻杆的横向振动阻尼; $F$ 为钻杆切削液 横向反馈力。

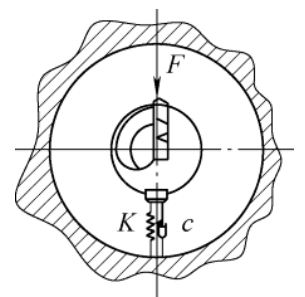

图 3 BTA 切削系统强迫振动模型

钻杆所受切削液反作用力可分解为径向力 $F_{e}$ 和 周向力 $F_{\varphi}$ 。从最大间隙处起沿钻杆转向取 $\theta$ 角为周 向坐标, 并考虑钻杆中心位置 $e, \varphi$ 的变化引起的切 削液厚度的变化, 利用 Reynolds 方程描述 ${ }^{[2]}$ 如下

$$
\begin{gathered}
\frac{1}{R^{2}} \frac{\partial}{\partial \theta}\left(\frac{h^{3}}{12 \mu} \frac{\partial p}{\partial \theta}\right)+\frac{\partial}{\partial Z}\left(\frac{h^{3}}{12 \mu} \frac{\partial p}{\partial Z}\right)= \\
\frac{1}{2}\left(\Omega-2 \frac{\mathrm{d} \varphi}{\mathrm{d} t}\right) \frac{\partial h}{\partial \theta}+\frac{\mathrm{d} e}{\mathrm{~d} t} \cos \theta
\end{gathered}
$$

式中 $R$ 一一钻杆半径;

$\Omega$ 一一钻杆旋转角速度;

$P$ 一一切削液压力分布;

$M$ 一一切削液的动力黏度;

$h$ 一一钻杆与工件内孔壁间的切削液厚度。其 中 $C$ 是钻杆与内孔壁间隙的平均值, 即 孔半径与钻杆半径之差;

$h=C(1+\varepsilon \cos \theta)$;

$\varepsilon$ 一钻杆的偏心率;

$\varepsilon=e / C, e=\overline{O O_{1}}$ 。

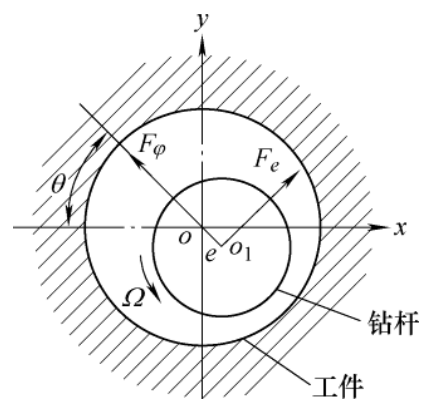

图 4 深孔切削系统 Reynolds 方程计算模型

文献[2]由式(21)简化整理并经过一系列计算， 得到切削液压力分布, 并求出钻杆所受流体作用力

$$
\begin{aligned}
& F_{e}=6 \mu R L\left(\frac{R}{C}\right)^{2}\left[\left(\Omega-2 \frac{\mathrm{d} \varphi}{\mathrm{d} t}\right) E_{1}(\varepsilon)+2 \dot{\varepsilon} E_{2}(\varepsilon)\right] \\
& F_{\varphi}=6 \mu R L\left(\frac{R}{C}\right)^{2}\left[\left(\Omega-2 \frac{\mathrm{d} \varphi}{\mathrm{d} t}\right) E_{3}(\varepsilon)+2 \dot{\varepsilon} E_{4}(\varepsilon)\right]
\end{aligned}
$$




$$
\begin{gathered}
E_{1}(\varepsilon)=\frac{2 \varepsilon^{2}}{\left(1-\varepsilon^{2}\right)\left(2+\varepsilon^{2}\right)} \\
E_{2}(\varepsilon)=\frac{1}{\left(1-\varepsilon^{2}\right)^{3 / 2}}\left[\frac{\pi}{2}-\frac{8}{\pi\left(2+\varepsilon^{2}\right)}\right] \\
E_{3}(\varepsilon)=\frac{\pi \varepsilon}{\left(1-\varepsilon^{2}\right)\left(2+\varepsilon^{2}\right)} \\
E_{4}(\varepsilon)=\frac{2 \varepsilon}{\left(1-\varepsilon^{2}\right)\left(2+\varepsilon^{2}\right)}
\end{gathered}
$$

当钻杆在偏心率最大位置时, 钻杆系统切削液 扰动的横向反馈力达到最大, $\varphi=0, \dot{\varepsilon}=\dot{\varphi}=0$ 整理得

$$
\begin{aligned}
& F_{e}=6 \mu R L\left(\frac{R}{C}\right)^{2} \Omega E_{1}(\varepsilon) \\
& F_{\varphi}=6 \mu R L\left(\frac{R}{C}\right)^{2} \Omega E_{3}(\varepsilon)
\end{aligned}
$$

在大长径比 BTA 深孔的精密扩孔加工中, 切削 余量很小, 横向切削力波动很小时, 钻杆横向激振 力波动值仅考虑切削液的扰动的反馈力波动值。

$$
F_{2}=F_{e}=6 \mu R L\left(\frac{R}{C}\right)^{2} \Omega E_{1}(\varepsilon)
$$

由式(24)以及图 5 不难看出, 切削系统切削液扰动 的切削液横向反馈力波动值 $F_{2}$ 与切削液黏度 $\mu$ 、钻 杆旋转角速度 $\Omega$ 以及钻杆的偏心率 $\varepsilon$ 有关。

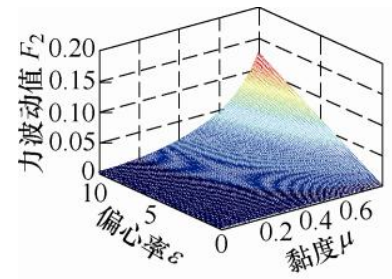

(a)

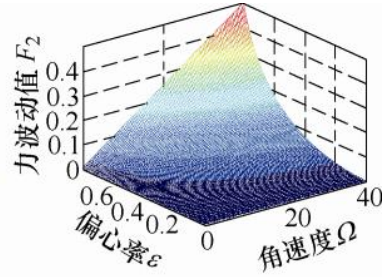

(b)
图 5 切削流体扰动的横向反馈力

\section{BTA 深孔切削系统横向振动的混} 沌解

在 BTA 深孔切削系统横向振动的研究中, 分 析系统的混沌特性必不可少。Melnikov 方法是研究 动力学混沌现象的基本解析方法。下面用此方法来 解析 BTA 深孔切削系统横向振动性态发生变化的 条件。

当系统未受扰动时, 式(20)的 Hamilton 函数为

$$
H(u, \dot{u})=\frac{1}{2} \dot{u}^{2}+\frac{\lambda}{4} u^{4}-\frac{\kappa}{2} u^{2}
$$

系统的相轨迹方程

$$
2 \dot{u}^{2}+\lambda u^{4}-2 \kappa u^{2}=4 c_{0}
$$

式中, $c_{0}$ 为任意常数。

得到相轨迹参数方程

$$
\begin{gathered}
u(t)= \pm \sqrt{\frac{2 \kappa}{\lambda}} \operatorname{sech}(\sqrt{\kappa} t) \\
\dot{u}(t)= \pm \sqrt{\frac{2 \kappa}{\lambda}} \sqrt{\kappa} \cosh (\sqrt{\kappa} t)
\end{gathered}
$$

将式(19)写成状态方程的形式如下

$$
f(u(t))=\left[\begin{array}{c}
\dot{u} \\
\kappa u-\lambda u^{3}
\end{array}\right]
$$

$$
\begin{gathered}
g(u, t)=\left[\begin{array}{c}
0 \\
-2 \eta \dot{u}+F_{1}+F_{2} \sin \left(\omega_{b} t\right)
\end{array}\right] \\
f(u(t)) \wedge g(u(t), t)=[-2 \eta \dot{u}(t)+ \\
\left.F_{1}+F_{2} \sin \left(\omega_{b} t\right)\right] u(t)
\end{gathered}
$$

将(29)、(30)代入 Melnikov 函数中得

$$
\begin{gathered}
M_{ \pm}(t)=\int_{-\infty}^{\infty}\left[-2 \eta \dot{u}^{2}(t)+F_{1} \dot{u}(t)+\right. \\
\left.F_{2} \sin \left(\omega_{b} t\right) \dot{u}(t)\right] \mathrm{d} t
\end{gathered}
$$

由式(31)解出系统发生横振的混沌阈值为

$$
\begin{gathered}
M_{ \pm}(t)=-\frac{16 \eta \kappa^{3 / 2}}{3 \lambda} \mp \sqrt{\frac{2}{\lambda}} \kappa F_{2} \pi \omega_{b} \bullet \\
\operatorname{csch}\left(\frac{\pi \omega_{b}}{2 \sqrt{\kappa}}\right) \sin \left(\omega_{b} t\right)
\end{gathered}
$$

由式(32)得到量纲一切削液扰动的横向反馈力 $F_{2}$ 应该满足条件

$$
F_{2}>\frac{16 \sqrt{\kappa} \eta}{3 \pi \omega_{b} \sqrt{2 \lambda}} \operatorname{sech}\left(\frac{\pi \omega_{b}}{2 \sqrt{\kappa}}\right)
$$

当 BTA 深孔切削系统的参数满足式(32)的条件 时, $M_{ \pm}(\tau)$ 必存在简单零点, 因此式(33)是 BTA 深孔 切削系统横向振动出现混沌现象的必要条件, 由图 (6)可知系统阻尼、刚度、外激励的变化, 都容易使 式(33)满足成立的条件, 即 BTA 深孔切削系统的结 构参数和工艺参数的变化都易使系统出现混沌。

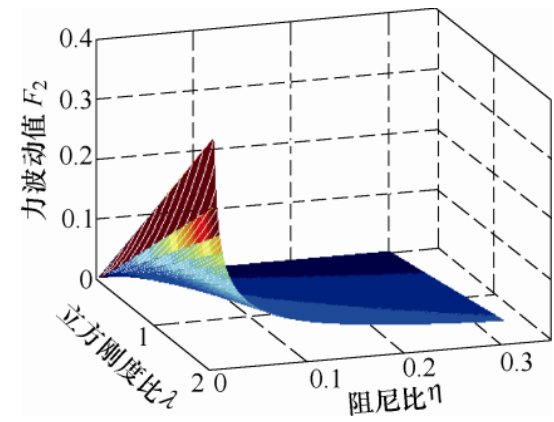


图 6 系统混沌现象必要条件图

\section{BTA 深孔切削系统横向振动的全局 吸引子}

BTA 深孔加工的高速精密扩孔过程中, 以加工 超临界汽轮机转子为例, 内孔 $D=50 \mathrm{~mm}$, 全长 $9.6 \mathrm{~m}$, 综合跳动 $5 \mu \mathrm{m}$, 表面粗糙度 $0.4 \mu \mathrm{m}$ 。选取各参数如 下: 钻杆总长 $L=20 \mathrm{~m}$, 钻杆外径 $d_{2}=46 \mathrm{~mm}$, 钻杆内 径 $d_{1}=30 \mathrm{~mm}$, 钻杆外切削液流速 $V_{1}=15 \mathrm{~m} / \mathrm{s}$, 钻杆最 大偏心率 $\varepsilon_{\max }=0.5$, 切削流体动力黏度 $\mu=10 \mathrm{~N} \cdot \mathrm{s} / \mathrm{m}^{2}$ 。 BTA 深孔钻杆的材料是锻制的高合金结构钢材料, $E=214 \times 10^{3} \mathrm{MPa}, \rho_{s}=7.78 \times 10^{3} \mathrm{~kg} / \mathrm{m}^{3}$, 切削液密度 $\rho_{f}=0.865 \times 10^{3} \mathrm{~kg} / \mathrm{m}^{3}, \quad c=61.2 \mathrm{~N} \cdot \mathrm{s} / \mathrm{m}, \quad k_{1}=3.7 \times 10^{4} \mathrm{~N} / \mathrm{m}$, $k_{3}=3.2 \times 10^{4} \mathrm{~N} / \mathrm{m}^{3}$ 。

系统参数量纲一化后得取值: $\eta=0.025, \kappa=0.5$, $\lambda=1.0, F_{1}=0.045, F_{2}=0.24$ 。为明确 BTA 深孔切削 动力系统混沌运动特性, 利用图胞映射法, 以量纲 一化后系统流体扰动的横向反馈力波动值 $F_{2}$ 为变 化参数, 对方程式(20)进行吸引子性态分析。

图 7a $7 \mathrm{f}$ 分别为 $F_{2}=0 、 0.06 、 0.08 、 0.19 、 0.26$ 、 0.36 时系统的吸引子、吸引域图。当 $F_{2}=0$ 时, BTA 深孔切削系统的相空间共存两个混沌吸引子 $\mathrm{B}(1)$ 、 $\mathrm{B}(2)$, 对应的吸引域位于同宿轨线内部区域, 此时 系统振子在同宿轨线内做小轨道运动如图 7a 所示。 随着扰动反馈力波动值 $F_{2}$ 增大, 混沌吸引子 $\mathrm{B}(1)$ 、 $\mathrm{B}(2)$ 逐渐增大, 并开始向外分散, 出现混沌吸引子 分形现象; 当 $F_{2}=0.06$ 时混沌吸引子 $\mathrm{B}(2)$ 和其所在 吸引域上的鞍点 1 发生碰撞, 系统发生第一次分岔; 当 $F_{2}=0.06 \sim 0.08$ 时, 从图 7b、图 7c 中可以看出两 个平衡点为中心的混沌吸引子开始融合, 并逐渐合 并成一个更大的混沌吸引子 $\mathrm{C}(1)$, 随着 $F_{2}$ 的继续增 大, 振子获得的能量也增加, 系统相空间出现周期 吸引子 $\mathrm{C}(2)$, 并与混沌吸引子 $\mathrm{C}(1)$ 共存, 分别存在 于各自的吸引域内, 且混沌吸引子 $\mathrm{C}(1)$ 和周期吸引 子 $\mathrm{C}(2)$ 都逐渐变大; 当 $F_{2}=0.19$ 时, 混沌吸引子 $\mathrm{C}(1)$ 与域边界上的鞍点 2 发生碰撞, 系统发生第二次分 岔, 如图 $7 \mathrm{~d}$ 所示。当 $F_{2}=0.26$ 时, 混沌吸引子 $\mathrm{C}(1)$ 与周期吸引子 (2) 融合, 并形成吸引子逃逸通道, 能 量高的混沌吸引子 $\mathrm{C}(1)$ 通过该通道逃出其所在吸引 域, 进入周期吸引子 $\mathrm{C}(2)$ 所在吸引域内, 如图 $7 \mathrm{e}$ 所示。当 $F_{2}=0.36$ 时, 混沌吸引子 $\mathrm{C}(1)$ 消失, 只剩 下周期吸引子 $\mathrm{C}(2)$ 做周期性运动如图 7f 所示。

对 BTA 深孔切削系统横向振动安全盆域的吸 引子、吸引域性态的分析明确了: 在 BTA 深孔精密
扩孔加工中, 系统在流体诱变下产生的类随机行为, 会导致该加工系统在流体诱变下发生混沌运动。

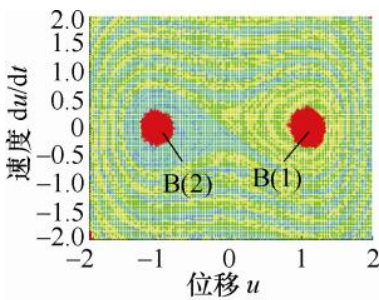

(a) $F_{2}=0$

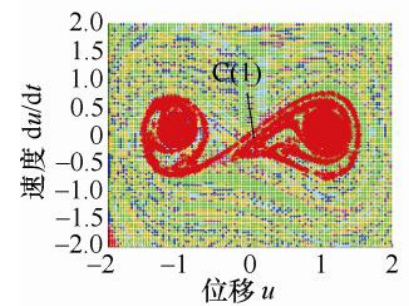

(c) $F_{2}=0.08$

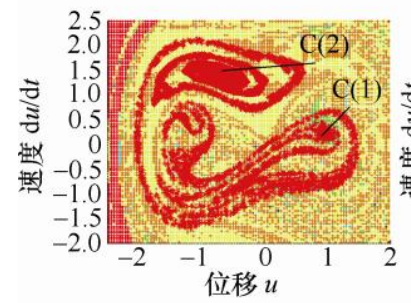

(e) $F_{2}=0.26$

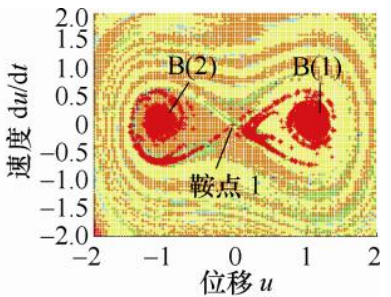

(b) $F_{2}=0.06$

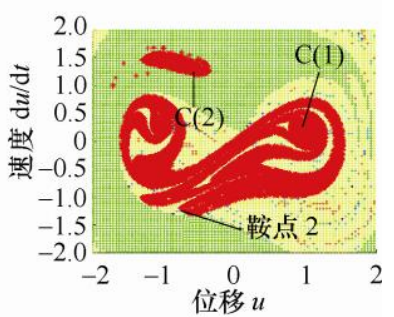

(d) $F_{2}=0.19$

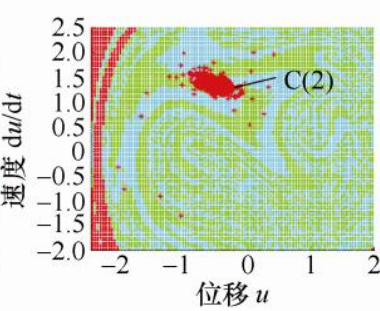

(f ) $F_{2}=0.36$
图 7 系统的吸引子、吸引域图

\section{BTA 深孔切削系统横向振动分贫}

为探究 BTA 深孔工艺系统中各参数变化对系 统横向非线性振动的影响机制, 取系统的量纲一参 数, 利用显式 Runge-Kutta 法仿真模拟方程式(20) 各参量变化时的分岔图与最大 Lyapunov 指数图。

以 BTA 深孔加工系统的阻尼比 $\eta$ 为分岔参数, 得到该系统的分岔图和最大 Lyapunov 指数, 如图 8 所示。由图 8 可见, 随阻尼比 $\eta$ 的变化, 系统出现 周期、混沌、Hopf 分岔等非线性动力特征。系统历 经单周期-混沌-概周期-混沌-概周期-二周期-单周期 的运动转迁。系统在阻尼比 $\eta=0.05$ 时, 发生分岔, 之后进入混沌运动; 当阻尼比变化到 $\eta=0.08$ 时, 系 统发生分岔, 转迁为概周期分岔; 当 $\eta=0.08 \sim 0.2$ 时, 


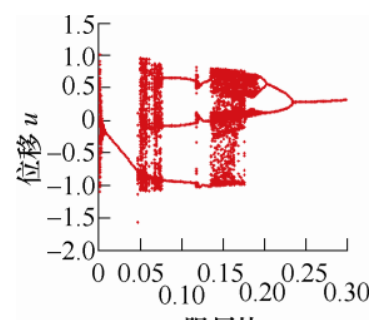

阻尼比 $\eta$

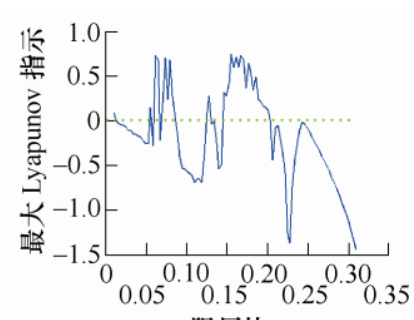

阻尼比 $\eta$

图 8 系统随 $\eta$ 变化的分岔图和最大 Lyapunov 指数图

系统再次进入混沌运动状态, 混沌运动状态系统的 最大 Lyapunov 指数大于零, 表明系统的非线性振动 强烈, 系统极不稳定。

图 9 为系统随立方刚度比 $\lambda$ 变化的分岔图。由 图 9 可见, 系统在 $\lambda=0 \sim 0.15$ 时呈单周期运动, 当 $\lambda=0.15$ 时, 系统发生分岔行为; 在 $\lambda=0.15 \sim 0.65$ 时 系统的最大 Lyapunov 指数在零以上频繁跳动, 系统 在周期、混沌状态频繁跳动切换, 不易控制; 当 $\lambda$ 大于 0.65 时, 系统的最大 Lyapunov 指数小于零, 系统转为稳定的单周期运动。

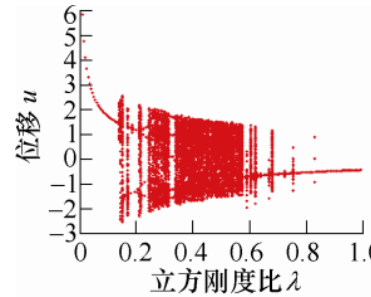

(a)

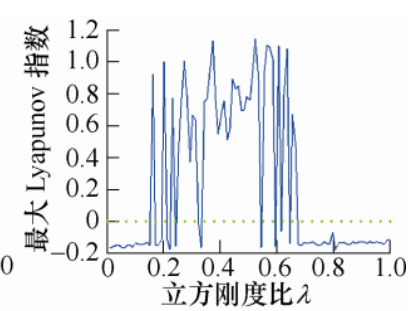

(b)

图 9 系统随 $\lambda$ 变化的分岔图和最大 Lyapunov 指数图

图 10 为系统随频率比 $\omega_{\mathrm{b}}$ 变化的分岔图。由图 10 可见, 系统经历混沌-单周期-混沌-概周期-单周 期的运动转迁。在 $\omega_{\mathrm{b}}=0 \sim 0.6 、 1.1 \sim 1.3$ 时系统的最 大 Lyapunov 指数大于零, 系统呈混沌运动状态。

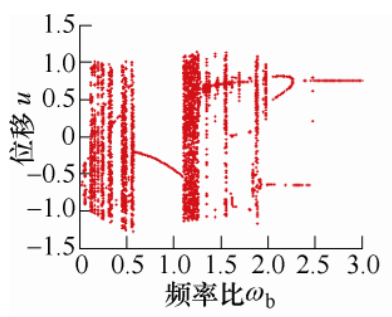

(a)

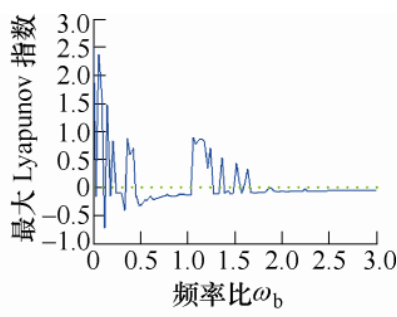

(b)

图 10 系统随 $\omega_{\mathrm{b}}$ 变化的分岔图和最大 Lyapunov 指数图

图 11 为系统随流体扰动的横向反馈力均值 $F_{1}$ 变化的分岔图。由图 11 可见, 系统在随 $F_{1}$ 变 化的过程中, 系统的最大 Lyapunov 指数始终小于 零, 系统在整个过程中呈现稳定的单周期运动。 说明在 BTA 深孔精密扩孔的加工过程中, 切削液 扰动的流体反馈力均值对系统的运动特性的改变 影响较小。
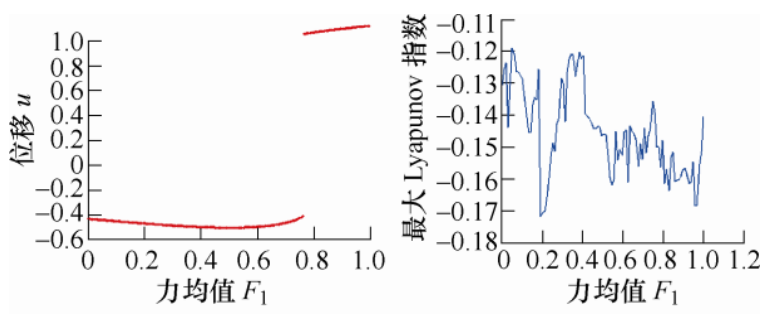

图 11 系统随 $F_{1}$ 变化的分岔图和最大 Lyapunov 指数图

图 12 为系统随切削液动力黏度 $\mu$ 变化的分岔图。 由图 12 可见, 在 $\mu=0 \sim 5$ 时系统最大 Lyapunov 指数 小于零, 呈单周期稳定运动; 当 $\mu=5$ 时, 系统发生 分岔行为, 之后进入混沌运动状态, 系统的最大 Lyapunov 指数大于零, 系统失稳; 当 $\lambda$ 变化到 11.5 时, 系统的最大 Lyapunov 指数小于零, 系统转为稳 定的周期运动。

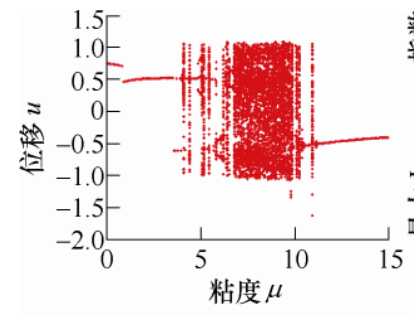

(a)

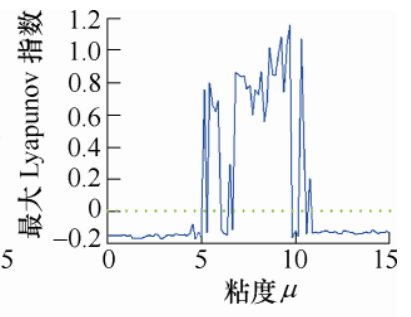

(b)
图 12 系统随切削液动力黏度 $\mu$ 变化的分岔图和 最大 Lyapunov 指数图

由图 13 可见, 在 $\Omega=0 \sim 10$ 时, 系统最大 Lyapunov 指数小于零, 呈单周期运动; 当 $\Omega=17$ 时, 系统发生分岔行为, 之后进入混沌运动状态, 系统 的最大 Lyapunov 指数大于零, 系统失稳; 当 $\Omega$ 变 化到 32 时, 系统的最大 Lyapunov 指数小于零, 系 统转为稳定的周期运动。

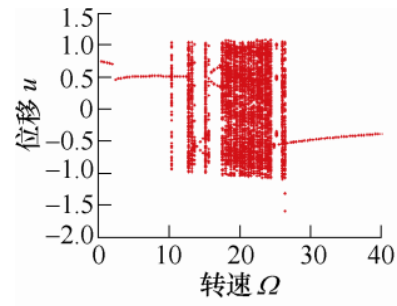

(a)

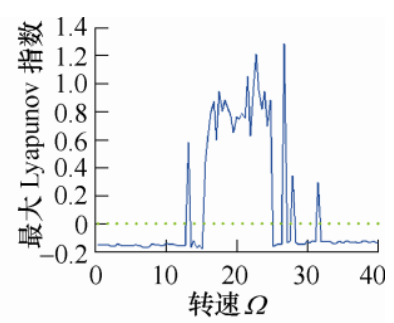

(b)
图 13 钻杆转速 $\Omega$ 变化的分岔图和最大 Lyapunov 指数图

\section{BTA 深孔切削系统横向振动双参} 特性

实际生产中, BTA 深孔加工系统往往被多个工 作参数施控, 其中系统的单参数变化随时可能引起 系统分岔, 导致运动性态发生变化。进一步考虑两 两匹配的参数同时变化对系统动力行为的影响, 可 
进一步明确双参数变化时系统的运动性态参数域。

图 14 为考虑系统立方刚度比 $\lambda$ 与系统阻尼比 $\eta$ 同时变化时, 系统在参数 $\eta-\lambda$ 平面上的动力学特性 参数域。图中 PA1 区域为系统最大 Lyapunov 指数 大于零的不稳定混沌区域; PA2 区域为系统最大 Lyapunov 指数在零附近的概周期或倍周期区域; PA3 区域为系统最大 Lyapunov 指数小于零的稳定的 单周期区域。参数域位于 PA1 区域内, 系统运动极 不稳定, 在生产中的参数选择应该尽量避开这样的 参数域。

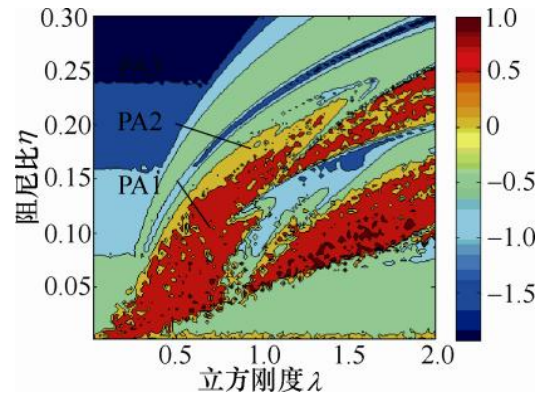

图 14 参数平面 $\eta-\lambda$ 上的分岔特性图

图 15 为考虑切削液动力黏度 $\mu$ 与系统阻尼比 $\eta$ 同时变化时, 系统在参数 $\mu-\eta$ 平面上的动力学特性 参数域。图中 PB1 区域为系统最大 Lyapunov 指数 大于零的不稳定混沌区域。PB2 区域为系统最大 Lyapunov 指数在零附近的概周期或倍周期区域。 PB3 区域为系统最大 Lyapunov 指数小于零的稳定 的单周期区域。参数域位于 PB1 区域内, 系统运动 极不稳定, 在生产中的参数选择应该尽量避开这样 的参数域。

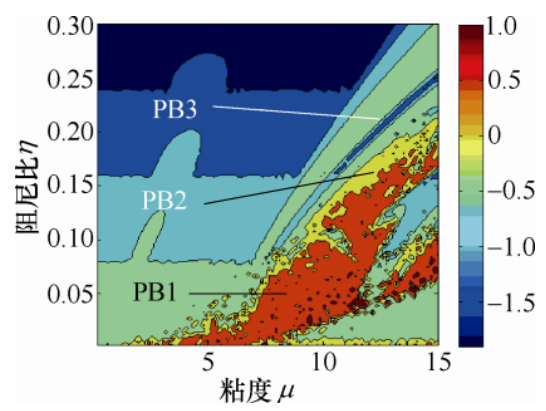

图 15 参数平面 $\mu-\eta$ 上的分岔特性图

图 16 为考虑切削液动力黏度 $\mu$ 与系统立方刚度 比 $\lambda$ 同时变化时, 系统在参数 $\mu-\lambda$ 平面上的动力学 特性参数域。图中 PC1 区域为系统最大 Lyapunov 指数大于零的不稳定的混沌区域。PC2 区域为系统 最大 Lyapunov 指数在零附近的概周期或倍周期区 域。PC1、PC2 区域构成了从周期到混沌变换的不 稳定带, PC3 区域为系统最大 Lyapunov 指数小于零 的稳定的单周期区域。在生产中的参数选择应该尽 量避开这一不稳定带。

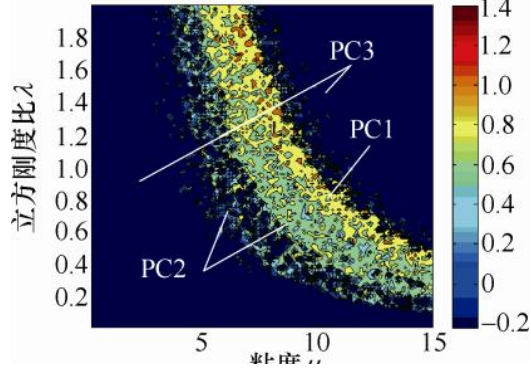

图 16 参数平面 $\mu-\lambda$ 上的分岔特性图

图 17 为考虑切削液动力黏度 $\mu$ 与系统激振力均 值 $F_{1}$ 同时变化时, 系统在参数平面 $\mu-F_{1}$ 上的动力学 特性参数域。图中 PD1 区域为系统最大 Lyapunov 指数大于零的不稳定混沌区域。PD2 区域为系统最 大 Lyapunov 指数在零附近的概周期或倍周期区域。 PD3 区域为系统最大 Lyapunov 指数小于零的稳定 的单周期区域。参数域位于 PD1 区域内, 系统运动 极不稳定，在生产中的参数选择应该尽量避开这样 的参数域。

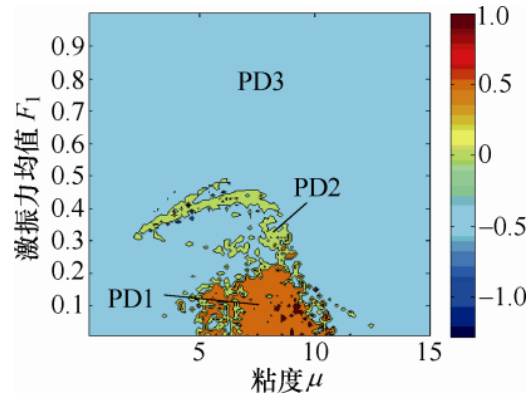

图 17 参数平面 $\mu-F_{1}$ 上的分岔特性图

图 18 为考虑切削液动力黏度 $\mu$ 与钻杆系统切 削流体扰动反馈力波动频率比 $\omega_{\mathrm{b}}$ 同时变化时, 系 统在参数 $\mu-\omega_{\mathrm{b}}$ 平面上的动力学特性参数域。图中 PE1 区域为系统最大 Lyapunov 指数大于零的不稳 定混沌区域。PE2 区域为系统最大 Lyapunov 指数 在零附近的概周期或倍周期区域。PE3 区域为系 统最大 Lyapunov 指数小于零的稳定的单周期区域。 参数域位于 PE1 区域内, 系统运动极不稳定, 在 生产中的参数选择应该尽量避开这样的参 数 域。

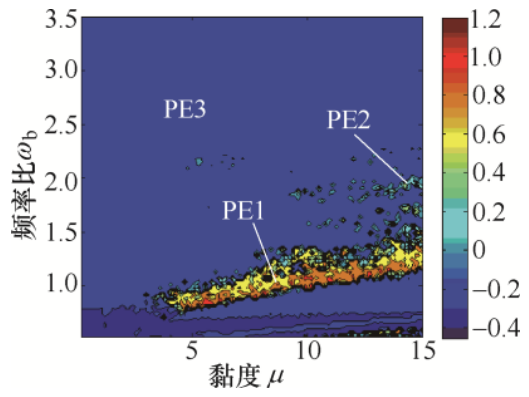

图 18 参数平面 $\mu-\omega_{\mathrm{b}}$ 上的分岔特性图 
图 19 和图 20 分别为切削液黏度 $\mu$ 与钻杆系统 切削液扰动波动的反馈力幅值 $F_{2}$ 、钻杆转速 $\Omega$ 同时 变化时的系统在参数 $\mu-F_{2}, \mu-\omega_{\mathrm{b}}$ 平面上的动力学特 性参数域。图中 PF1、PG1 区域为系统最大 Lyapunov 指数大于零的不稳定的混沌区域, 此时, 系统的运 动状态极不稳定, 容易失稳, 在生产中的参数选择 应该尽量避开这样的参数域。

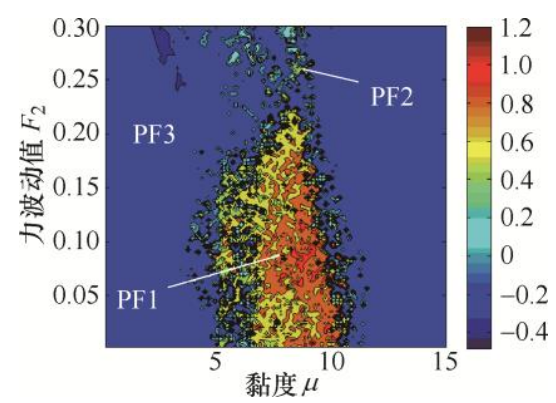

图 19 参数平面 $\mu-F_{2}$ 上的分岔特性图

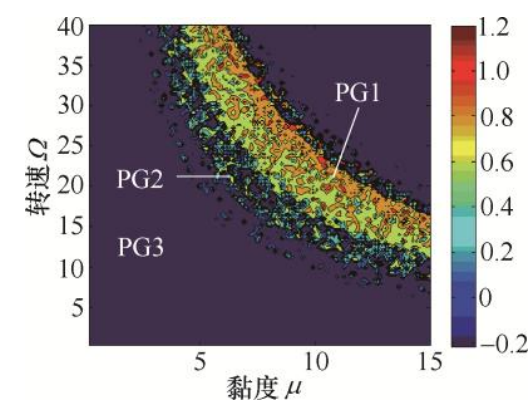

图 20 参数平面 $\mu-\Omega$ 上的分岔特性图

\section{6 结论}

本文以 BTA 深孔切削系统为研究对象, 建立了 切削液流体扰动下 BTA 深孔切削系统横向非线性 振动模型, 结合分岔和最大 Lyapunov 指数等特征, 分析了流体扰动激励下 BTA 深孔切削系统随各类 参数变化的非线性动力行为, 通过双参平面分岔分 析, 讨论了该系统在双参变化时的非线性动力特性。 通过对 BTA 深孔加工系统安全盆域中吸引子和吸 引域的流形变化, 明确了在多初值条件下, 该系统 吸引子的运动特性。结论如下。

(1) 考虑多初值对 BTA 深孔加工系统非线性振 动特性的影响, 在多初值条件下会出现混沌吸引子 增大、分形以及混沌吸引子间的共存、融合的现象。

(2) 在阻尼比 $\eta$ 、三次方刚度比 $\lambda$ 、切削液黏度 $\mu$, 流体力波动频率 $\omega_{\mathrm{b}}$ 变化时, 系统会出现周期、 混沌交替出现的运动特性, 利用仿真的结果, 可避 免实际生产中系统的非线性不可控振动。

(3) 获得了系统三次方刚度比-系统阻尼比、 切削液动力黏度-系统阻尼比、切削液动力黏度-
系统三次方刚度比等 7 组双参数变化时, 出现不 稳定混沌状态对应的双参数域, 可为实际生产控 制提供参考

通过对 BTA 深孔切削系统非线性动力行为及 运动路径的研究, 明确了该系统在流体扰动激励下, 系统的工作状态并不稳定, 流体的作用效果并不唯 一, 或促进系统稳定, 或促进系统失稳。在实际生 产中应尽量避开发生混沌运动的参数域。

\section{参 考 文 献}

[1] 胡占齐, 赵武, 缪否. BTA 深孔加工中流体力引起的钻 杆浴动的研究[J]. 机械工程学报, 2005, 41(1): 230-233. HU Zhanqi, ZHAO Wu, MIAO Lei. Research on vortex motion of BTA drilling shaft caused by hydro-force[J]. Journal of Mechanical Engineering, 2005, 41(1): 230-233.

[2] 赵武. BTA 深孔钻杆的力学行为研究[D]. 秦皇岛: 燕山 大学, 2003.

ZHAO Wu. Study on the mechanics behavior of BTA deep hole drill shaft[D]. Qinhuangdao : Yanshan University, 2003.

[3] 贾伟涛. BTA 深孔钻杆的动态特征及稳定性分析 [D]. 焦作: 河南理工大学, 2014.

JIA Weitao. Dynamic characteristics and stability analysis on BTA deep-hole boring bar[D]. Jiao Zuo: Henan Polytechnic University, 2014.

[4] ZHAO Wu, CHEN Dejie, HU Zhanqi. Center track analysis and simulation considering effect of vortex and perturbation of cutting fluid on BTA boring $\operatorname{bar}[\mathrm{J}]$. Applied Mechanics \& Materials, 2014, 526: 150-154.

[5] ZHAO Wu, CHEN Dejie. Research on dynamic fatigue strength criterion of BTA deep hole boring bar for consideration vortex surrounded by internal and external cutting fluid[J]. Advanced Materials Research, 2011, 189-193: 2264-2268.

[6] 罗忠, 朱云鹏, 韩清凯, 等. 动力学相似理论及在结构 振动分析中的应用研究评述与展望[J]. 机械工程学报, 2016, 52(23): 114-134.

LUO Zhong, ZHU Yunpeng, HAN Qingkai, et al. review and prospect for dynamic similitude theory and its applications in the structure vibration[J]. Journal of Mechanical Engineering, 2016, 52(23): 114-134.

[7] 丁虎, 陈立群. 轴向运动梁横向振动非线性模型数值研 究 $[C] / /$ 全国非线性动力学和运动稳定性学术会议, 2009.

DING Hu, CHEN Liqun. A numerical investigation into nonlinear models for transverse vibration of beams [C]//. National Conference on Nonlinear Dynamics and Stability of Motion, 2009. 
[8] 韦娟, 宁方立, 郭琪否, 等. 谐振管内非线性驻波的间 断 Galerkin 方法[J]. 机械工程学报, 2016, 52 (23): 141-151.

WEI Juan, NING Lifang, GUO Qilei, et al. Discontinuous Galerkin method for nonlinear standing waves in acoustic resonators[J]. Journal of Mechanical Engineering, 2016, 52(23): 141-151.

[9] 金基铎, 秦朝红, 李大伟. 轴向流中细长圆柱体的简化 模型及动态特性分析 [J]. 振动工程学报, 2011, 24 (1): 26-30.

JIN Jiduo, QIN Zhaohong, LI Dawei. A simplified model for a slender flexible cylinder subjected to axial flow and its dynamic characteristics[J]. Journal of Vibration Engineering, 2011, 24 (1): 26-30.

[10] XU W, HE Q, FANG T. Global analysis of stochastic bifurcation in duffing system[J]. International Journal of Bifurcation and Chaos, 2003, 13(10): 3115-3123.

[11] 徐伟, 贺群, 戎海武, 等. Duffing Van der Pol 振子随机 分岔的全局分析 [J]. 物理学报, 2003, 52(6)：1265-07. XU Wei, HE Qun, RONG Haiwu, et al. Global analysis of stochastic bifurcation in a buffing van der Pol system[J]. Acta Physica Sinica, 2003, 52(6): 1265-07.

[12] 史蒂芬 H. 斯托加茨. 非线性动力学与混沌[M]. 北京: 机械工业出版社, 2016.

STEVEN H S. Nonlinear dynamics and chaos[M]. Beijing: China Machine Press, 2016.

[13] 徐伟. 非线性随机动力学的若干数值方法及应用 $[\mathrm{M}]$. 北京: 科学出版社, 2013.

$\mathrm{XU}$ Wei. Numerical analysis methods for stochastic dynamical system[M]. Beijing: Science and Technology Press, 2013.

[14] BYKADOR V S, BYKADOR Z E. Bifurcations of deep hole drilling process[J]. Procedia Engineering, 2017, 206: 151-156.

[15] CHEN Jinhua, SHEU Shoude. Strengths and weaknesses of finite element modeling deep hole drilling as compared with beam and column equations[J]. Int. J. Adv. Manuf. Technol., 2007, 32: 229-237.

[16] WEINERT K, WEBBER O, PETERS C. On the influence of drilling depth dependent modal damping on chatter vibration in bta deep hole drilling[J]. CIRP Annals Manufacturing Technology, 2005, 54(1): 363-366.

[17] 张洁, 刘成颖, 郑烽, 等. 基于铣削动力学的刀具强迫 振动抑制研究[J]. 机械工程学报, 2018, 54(17): 94-99. ZHANG Jie, LIU Chengying, ZHENG Feng, et al. Research on suppression of the forced vibration of the cutter based on the milling dynamics[J]. Journal of Mechanical Engineering, 2018， 54 (17): 94-99.

[18] 孔令飞, 孙理论, 刘瑶, 等. BTA 深孔钻削过程切削热 通量的追踪方法研究 [J]. 机械工程学报, 2018,54(15): 213-220.

KONG Lingfei, SUN Lilun, LIU Yao, et al. Tracing algorithm of workpiece heat flux in deep hole processing[J]. Journal of Mechanical Engineering, 2018, 54(15): 213-220.

作者简介: 赵武, 男, 1971 年出生, 博士, 副教授, 博士研究生导师。 主要研究方向为非线性动力学、精密超精密加工技术与装备。

E-mail: zhaowu@hpu.edu.cn

霍博义, 男, 1990 年出生。主要研究方向为非线性动力学、精密超精密 加工技术与装备。

E-mail: huoboyi@126.com

黄丹(通信作者), 女, 1978 年出生, 博士, 副教授, 硕士研究生导师。 主要研究方向为高性能金属及金属基复合材料、抗冲击防护材料。

E-mail: huangdan@hpu.edu.cn 